\title{
Multi-party referential communication in complex strategic games
}

\author{
Jessica Mankewitz*1, Veronica Boyce*1 \\ Brandon Waldon' ${ }^{2}$, Georgia Loukatou ${ }^{1}$, Dhara $\mathrm{Yu}^{3}$ \\ Jesse $\mathrm{Mu}^{3}$, Noah D. Goodman ${ }^{1,3}$, Michael C. Frank ${ }^{1}$ \\ ${ }^{1}$ Department of Psychology, ${ }^{2}$ Department of Linguistics, ${ }^{3}$ Department of Computer Science \\ Stanford University \\ \{jmankewitz, vboyce, bwaldon, loukatou, dharakyu, muj, ngoodman, mcf rank\}@stanford. edu
}

\begin{abstract}
Verbal communication is an ubiquitous aspect of human interaction occurring in many contexts; however, it is primarily studied in the limited context of two people communicating information. Understanding communication in complex, multi-party interactions is both a scientific challenge for psycholinguistics and an engineering challenge for creating artificial agents who can participate in these richer contexts. We adapted the reference game paradigm to an online 3-player game where players refer to objects in order to coordinate selections based on the available utilities. We ran games with shared or individual payoffs and with or without access to language. Our paradigm can also be used for artificial agents; we trained reinforcement learning-based agents on the same task as a comparison. Our dataset shows the same patterns found in simpler reference games and contains rich language of reference and negotiation.
\end{abstract}

\section{Introduction}

Communication is an ubiquitous aspect of human interaction, allowing information sharing, creative expression, and coordination with other people. Classic and current approaches to communication largely assume dyadic interactions with a goal of information transfer [1, 2]. Our broad aim here is to expand our understanding of communication to multi-party interactions with a broader set of goals. We do so by exploring communication in complex, partial-knowledge three-player games.

Communication is often conceptualized as a process of mentalistic reasoning about others' intentions, given their goals [1]. Often the sole goal is information transfer, but not always. For example, people routinely produce polite language that reflects trade-offs between informativity and social goals like kindness [3]. How is language use modulated by the mix of cooperative and competitive goals that characterize many real-world interactions?

We focus on object reference, which has been a key case study for understanding how interlocutors collaborate using dialogue. In iterated reference games, a speaker repeatedly describes images from a tableau for a listener to select. Over iterations on the same images, the speaker's utterances reduce in length as the two converge to a shorthand [4]. This "reduction" phenomenon is a key signature for efficient communication. The games can be conducted over the web using chat boxes as the interface [5], providing a scalable environment for studying collaborative reference. While most of this literature focuses on two-party reference games, similar patterns of reduction were found when one speaker instructed multiple listeners [6].

The use of artificial agents provides a method for making comparisons between human behavior and behaviors resulting from a known set of (often normative) assumptions or principles. For

Preprint. Under review. 


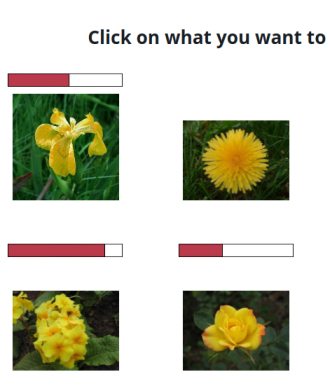

(a) Selection

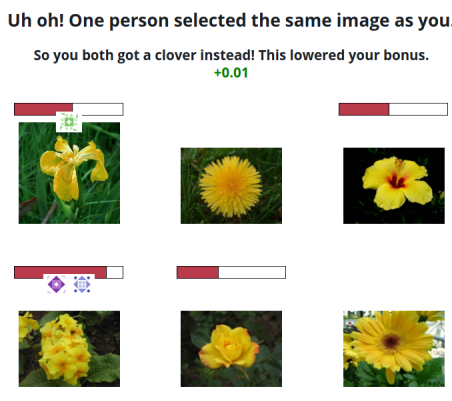

(b) Feedback

\begin{tabular}{c|c|c} 
& Indiv. & Shared \\
\hline P 1 & 2 & 3.66 \\
P 2 & 7 & 3.66 \\
P 3 & 2 & 3.66
\end{tabular}

(c) Value of selections

Figure 1: In the selection phase (a), each player sees 6 flowers and the value bars of 4 of them. In the feedback stage (b), players see who chose what and how much they earned. Here, $\mathbf{P} \mathbf{1}$ and $\mathbf{P} \mathbf{3}$ both selected the 11-value flower, but they instead get clover ( 2 points) because of the multiple-selection. $\mathbb{P} 2$ is the only one to select the 7-value flower, so they earn 7 points. In the shared condition, points are averaged, for 3.66 points per player.

example, the "rational speech acts" approach determines the interpretation of a speakers' utterances via recursive mental modelling starting from the literal extensions of words and the current context [2]. This framework builds on previous game-theoretic approaches [7], formalizing the idea of communication as optimizing a utility function where the greatest reward comes from the perfect transfer of knowledge. When agents developed in this tradition are placed in iterated reference games, they show some adaptation to human speakers [5], but their accuracy is lower than human listeners.

In the current work, we extend the reference game paradigm to groups of three players playing a game with a more complex utility structure. To optimally coordinate and negotiate their selections, participants must make repeated references to hard-to-name images in varying contexts. This communication environment relaxes the rigid structure of classic reference games and allows more naturalistic interaction. We make use of this paradigm to measure human behavior across different conditions (varying both the payoffs of the game and the ability to chat with other players). Because the structure of our game is complex, we were interested in the maximal available reward for agents coordinating with or without language; to estimate these rewards we trained reinforcement learning agents on the same games that humans played, allowing them in some conditions to invent signaling systems for the purpose of coordination.

\section{Human Experiment}

Procedure We recruited triads of participants to play a real-time coordination and communication game implemented using the Empirica platform [8]. Participants first gave informed consent and then proceeded at their own pace through a set of introduction slides. The game was framed as sharing a farm where they ordered plants from Farmer Bob. They were told Farmer Bob made 6 plants available each month, which varied in profitability. If multiple people selected the same flower, they each would receive clover, a low-profit plant, instead. Players had to pass a series of short comprehension quizzes as they went through the introduction to ensure they understood the game mechanics. Then players waited in a lobby until others were ready.

Each game consisted of a total of 24 trials using 12 flower images [9] that reoccurred randomly across trials. Flowers were selected to provoke complex referring expressions; within each game, flowers were similar in color, but dissimilar in shape and texture (Fig. 1a). On every trial, players saw a set of 6 flowers on the screen, with display order randomized between players. Each flower was assigned an integer reward value; one of the values was drawn uniformly from 10-12, and the rest were drawn without replacement from 1-9 (guaranteeing one high value flower). Each player saw the values represented as colored bars (Fig. 11) for 4 of the 6 flowers; the value of each flower was hidden from exactly one player. In the selection phase (Fig. 1a, players each clicked on a flower; once clicked, selections could not be changed. Once all players had selected (or the 3 minute timer had elapsed), players where shown feedback (Fig. 1b) on who selected what and how much they 
had earned. If only one player selected a flower, their selection was worth the value of the flower in points; if multiple people selected the same flower, each selection was worth 2 points (Fig. 1c).

During the game, the cumulative earnings of the player and their partners were displayed on the side of the screen. At the end of the game, players were each asked how they would describe the flower images they played with (as well as some control images). Finally, participants were asked for optional demographic information and feedback on their experience.

We varied both the utility structure (individual vs. shared) and the availability of language (language vs. no-language) in a $2 \times 2$ crossed design. In the individual condition, players earned points for what they selected. In the shared condition, points were pooled and they each earned the average of what each would have gotten in the individual condition. In the language condition, players had free access to a chat box; in the no-language condition, the chat box was disabled.

Participants We recruited 261 participants from Prolific. Participants were paid $\$ 3.33$ - \$5.00 dollars and up to $\$ 3.30$ in earned bonuses for an average hourly compensation of $\$ 18.19$ USD (\$1943.67 USD total). We report results from 18 complete individual language games, 21 shared language games, 20 individual non-language games, and 18 shared non-language games. We excluded 13 games that ended when a participant disconnected 1

\section{Reinforcement Learning Agents}

We trained deep reinforcement learning (RL) agents to play the same games. We represented images by unique identifiers (one-hot vectors) rather than pixel values, to focus on the interaction dynamics rather than language for complex visual stimuli. Agents were trained to optimize their points earned. Groups of agents were assigned to the same $2 \times 2$ set of conditions tested with humans.

For the "language" conditions, we provided agents a communication channel in order to explore how such a channel could support coordination (Cf. work on emergent language, [10]). Each agent encoded their visible game state (items + utilities) with a Transformer encoder [11]. Agents then participated in one round of dialogue: they generated messages, represented as real-valued, 20dimensional vectors, conditioned on their encoders' pooled output; they exchanged these messages, and re-encoded the game state with the dialog context; they then generated a final decision. We trained agents over $1.6 \times 10^{6}$ games, running 10 trials for each condition; see Appendix for model and training details.

\section{Results}

The language conditions of our games produced a rich corpus ( $25 \mathrm{~K}$ words from $\sim 120$ speakers) which has been checked for harmful or offensive language. Amount of language produced, game length, and points earned were all positively correlated (Fig. 2A). No-language groups generally earned low rewards and finished the games within 5-10 minutes. Groups in the language condition varied in how much they used the chat box: 4 of the groups said nothing, while others produced up to $\sim 2,000$ words. Across groups, an apparent Pareto frontier emerged, with greater language use associated with greater group earnings, but also with longer game length, reflecting a trade-off between speed and earnings.

Language use declined over the course of games (Fig. $2 \mathrm{~B}$ ), consistent with the hypothesis that they were reducing the length of their referring expressions. In addition, average rewards increased modestly over games $(r=.09, p=.01)$. Later in the game, participants used fewer words to achieve the same or greater utility, indicating that they were achieving greater communicative efficiency. Further, participants in the individual utility condition tended to use more words overall. One speculation is that, in addition to sharing information, groups in the individual utility condition may have needed to do more negotiation about how to share rewards.

The RL agents performed better than humans across all conditions, but this comparison is difficult to interpret. The RL agents are efficient statistical learners that are able to take advantage of regularities in the structure of the game (e.g., if the four flowers that are available are all low in value, the

${ }^{1}$ Our experimental code and instructions, data, and analysis code are all available at https://osf.io/ 3kudj/?view_only=0c043c5a678940919a06c8c3cc05bc80 
A

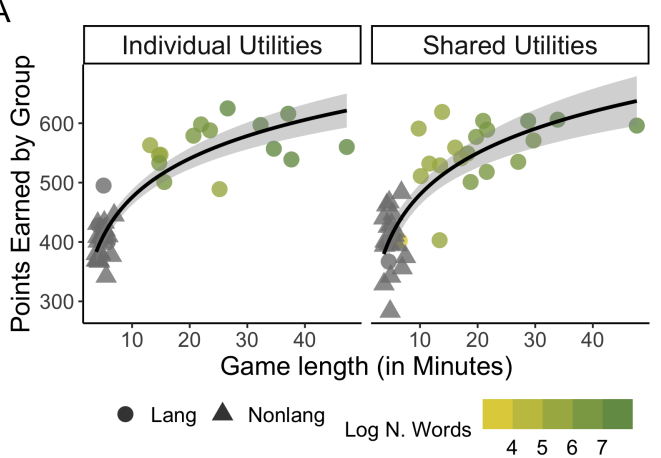

B

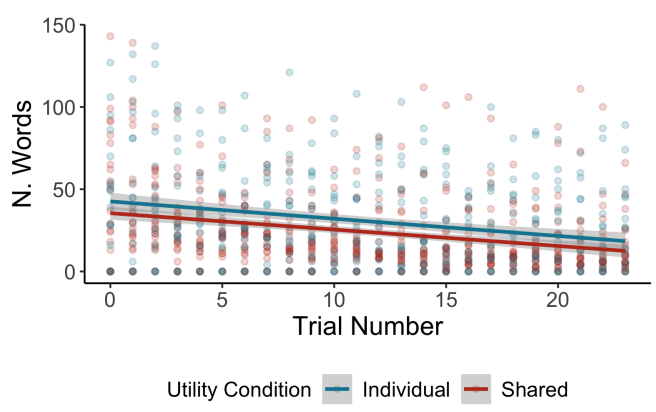

Figure 2: (A) Total points earned in each game, plotted by game length and utility condition. Each point is a game, with color denoting amount of language use (gray = no language use) and shape denoting language/no-language condition. (B) Number of words per trial plotted by trial number. Each point represents one game.

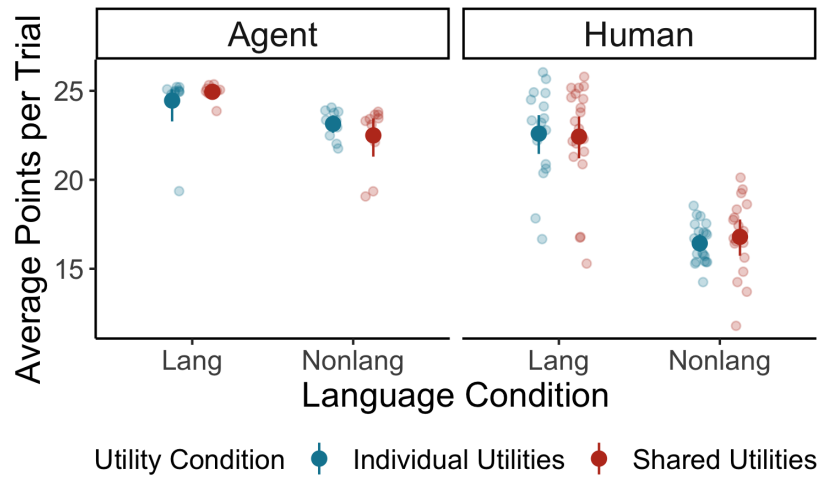

Figure 3: Average points earned per trial, summed over of the players in each game. Dots show individual games, larger points are mean and $95 \%$ confidence intervals.

flowers with hidden value information are probably high in value); they are also trained on orders of magnitude more trials than the human agents.

Using language provided a benefit to both humans and RL agents (Fig. 3), but the benefit was much larger for humans. By declaring their intentions and sharing information (e.g., the values of flowers that their partners can't see), communicating helps human players maximize their profit. Communication benefited the RL agents as well, but to a lesser extent. This lower benefit likely reflects the better performance achieved without communication, but perhaps also the greater flexibility of human language for sharing relevant values and strategies.

\section{Discussion}

Humans communicate across a vast range of different contexts, many of which are not dyadic exchanges of information between perfectly cooperative speakers. We introduced a new game paradigm that builds on the classic reference game but adapts it to a 3-party coordination and negotiation framework. In this new context, we still find the classic pattern of increasing efficiency in communication, but also see trade-offs between using language to optimize rewards vs. coordinating more quickly without language. We also demonstrated a set of artificial agents with emergent language and behaviors that allow us to compare the utility of language for coordinating their behaviors to the utility it provides for human players.

The language produced during these games is rich with negotiation, intention declarations, and complex image descriptions, making them a resource for future analyses of human pragmatics as well as a resource for training agents to use pragmatic language. Further, these games are a first step towards an empirical framework for exploring communication in multi-party contexts. Our game 
structure can be easily adapted to different numbers of players, configurations of knowledge states, and arbitrarily complex reward structures. We hope this work will help us better understand the relationship between joint coordination and communication.

\section{Acknowledgments and Disclosure of Funding}

This project was supported by the HAI Hoffman-Yee grant. We would also like to thank Chris Potts, Mariano-Florentino Cuéllar, Dorsa Sadigh, and Judith Degen for their helpful discussions.

\section{References}

[1] Herbert P Grice. Logic and conversation. In Speech acts, pages 41-58. Brill, 1975.

[2] Noah D. Goodman and Michael C. Frank. Pragmatic Language Interpretation as Probabilistic Inference. Trends in Cognitive Sciences, 20(11):818-829, November 2016.

[3] Erica J. Yoon, Michael C. Frank, Michael Henry Tessler, and Noah D. Goodman. Polite speech emerges from competing social goals. Preprint, PsyArXiv, December 2018.

[4] Herbert H Clark and Deanna Wilkes-Gibbs. Referring as a collaborative process. Cognition, 1986.

[5] Robert D. Hawkins, Minae Kwon, Dorsa Sadigh, and Noah D. Goodman. Continual adaptation for efficient machine communication. arXiv:1911.09896 [cs], October 2020.

[6] Si On Yoon and Sarah Brown-Schmidt. Audience Design in Multiparty Conversation. Cognitive Science, 43(8):e12774, 2019.

[7] Gerhard Jäger. 93. game theory in semantics and pragmatics. In Volume 3, pages 2487-2516. De Gruyter Mouton, 2012.

[8] Abdullah Almaatouq, Joshua Becker, James P. Houghton, Nicolas Paton, Duncan J. Watts, and Mark E. Whiting. Empirica: A virtual lab for high-throughput macro-level experiments. arXiv:2006.11398 [cs], December 2020.

[9] Maria-Elena Nilsback and Andrew Zisserman. A visual vocabulary for flower classification. In IEEE Conference on Computer Vision and Pattern Recognition, volume 2, pages 1447-1454, 2006.

[10] Angeliki Lazaridou and Marco Baroni. Emergent multi-agent communication in the deep learning era, 2020.

[11] Ashish Vaswani, Noam Shazeer, Niki Parmar, Jakob Uszkoreit, Llion Jones, Aidan N. Gomez, Łukasz Kaiser, and Illia Polosukhin. Attention is all you need. In Proceedings of the 31st International Conference on Neural Information Processing Systems, 2017. 


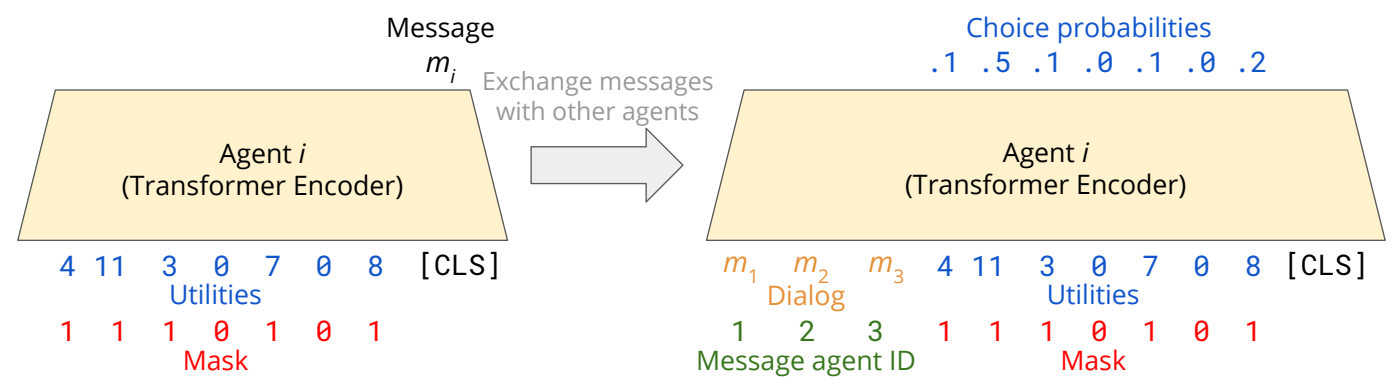

Figure S1: Agent architecture.

\section{A Models and training details}

Link to code and data used to reproduce the agent experiments is available at https://anonymized

\section{Models}

Each agent (Figure S1) consists of a 2-layer Transformer encoder with hidden size 64 and 8 attention heads. Each game is represented as a sequence of 6 utilities ( 0 if the utility is unknown) as well as a supplemental binary mask feature which indicates whether the utility is known to the agent. An agent encodes the game state (represented by utilities and mask features) along with a special [CLS] token with its Transformer. The encoded [CLS] token is then converted to a 20-dimensional message with a single linear layer, then sent to the other agents. Finally, the agent takes all agent messages generated during a round of dialog, along with indices corresponding to which agent produced each message, and concatenates these features with the original game state. This updated game state is then re-encoded with the same transformer ${ }^{2}$ The encoded states for each game item are then converted via a linear layer to a distribution over corresponding selection actions in the game.

The agents are end-to-end differentiable due to the use of a continuous communication protocol. We computed expected reward for each of the agents' possible decisions, then maximized this expected reward for each agent with respect to its parameters via gradient descent.

\section{Training}

Models were trained with the Adam optimizer and learning rate 5e-5. They were trained for 500 epochs with step size 100 and batch size 32, on several Nvidia Titan X Pascal GPUs which are part of an internal cluster. Each run took approximately 2 hours. We procedurally sample games during training; the number of games seen throughout a single training run is $1.6 \times 10^{6}$.

\footnotetext{
${ }^{2}$ Note this paradigm allows straightforward extension to multiple rounds of dialog, where messages are re-generated and re-encoded using the same transformer.
} 